\title{
Llaqtaqasa, un asentamiento del Periodo Intermedio Tardío (1100-1400 d. C.) ubicado en el distrito de Concepción, provincia de Vilcas Huamán (región Ayacucho)
}

\author{
Winnie Celeste Martínez Sulca \\ Universidad Nacional Mayor de San Marcos \\ wicemar-271193@hotmail.com
}

\section{RESUMEN}

En este artículo presentaremos, a grandes rasgos, el sitio arqueológico Llaqtaqasa ubicado en el distrito de Concepción, provincia de Vilcas Huamán. Dentro de esta se describirán los sectores que la conforman, las estructuras arquitectónicas aledañas y los materiales arqueológicos muebles hallados en superficie. Con el posterior análisis de estos datos planteamos que Llaqtaqasa fue construido durante el Periodo Intermedio Tardío en un punto estratégico conformado por grandes áreas de pampas asociadas a fuentes de agua donde desarrollaron actividades productivas.

Palabras clave: Llaqtaqasa, Periodo Intermedio Tardío, Vilcas Huamán

\section{Llaqtaqasa, a settlement of the Late Intermediate Period (1100- 1400 AD) located in the district of Concepción, Vilcas Huamán province (Ayacucho region)}

\begin{abstract}
In this article, we will present, in grosso modo, the Llaqtaqasa archaeological site in the district of Concepción, province of Vilcas Huamán. Within this site, we will describe the sectors comprising it, the surrounding architectural structures, and the movable archaeological materials found on the surface. By analyzing this data, we propose a building date during the Late Intermediate Period, in a strategic point made up of large pampa areas with water sources used for productive activities development.
\end{abstract}

KeYwords: Llaqtaqasa, Late Intermediate Period, Vilcas Huamán 


\section{Introducción}

E sitio arqueológico Llaqtaqasa es uno de los pocos sitios emplazados sobre uno de los puntos más altos dentro de la provincia de Vilcas Huamán, a una altura que va de 4000 a $4150 \mathrm{msnm}$, tiene un dominio visual total del área circundante y desde este sitio se puede observar el río Pampas (actual límite con la región de Apurímac), el apu Ccarhuarazo (provincia de Lucanas), el nevado Salkantay (región Cusco), entre otros. Además, en los alrededores hemos registrado grandes áreas de pampas, fuentes de agua y abrigos rocosos que hicieron posible el desarrollo de actividades productivas como la crianza de camélidos y la agricultura de altura. Esto último se ve evidenciado por la presencia de andenes y corrales ubicados dentro y en los alrededores del sitio.

El único antecedente de investigación de este asentamiento son los trabajos de prospección realizados por Ochante (2001), quien lo clasificó como un sitio de habitación conformado por estructuras circulares asociados a corrales pertenecientes al Periodo Intermedio Tardío. Debido a que no se cuenta con más información es que se decidió caracterizar arquitectónicamente el asentamiento y ubicarlo cronológicamente a partir de la evidencia expuesta en superficie.

En este asentamiento realizamos varios trabajos de campo que incluyeron prospección, levantamiento planímetrico y topográfico, análisis de materiales insitu, análisis de la arquitectura en superficie, entre otros. Esto como parte de la tesis de licenciatura de la Universidad Nacional Mayor de San Marcos que está próxima a sustentarse.

Producto de este trabajo se realizó la sectorización en 3 áreas claramente diferenciadas donde el sector $\mathrm{A}$ y $\mathrm{B}$ corresponderían al área doméstica, siendo el sector B el más extenso con un mayor número de recintos circulares, además de estar conformada por tres muros perimetrales. Por su parte, el sector C estaría conformado por una serie de corrales asociados a recintos circulares, todos estos destinado a la crianza de camélidos.

En base a las características arquitectónicas, los componentes, el emplazamiento y los materiales hallados en superficie, como la cerámica del grupo Campanayuq marrón rojizo alisado simple (Mendoza, 2010) el tipo Arqalla y Tanta Orqo (González et al,
1987), podemos mencionar que el sitio arqueológico Llaqtaqasa fue construido durante el Periodo Intermedio Tardío y probablemente durante el Periodo Horizonte Tardío fue abandonado. Además, los recintos circulares al interior del sector B presentan una organización espacial denominado "Grupos-patio", definido para la sierra central por T-Earle (1987).

\section{Antecedentes en torno al Periodo Intermedio Tardío en Vilcas Huamán}

Lumbreras (1959), entre los años 1956 a 1958 inició sus exploraciones en la sierra central, trató de definir la existencia de los grupos denominados chancas, mencionados en las crónicas, para el área de Ayacucho. En el distrito de Vilcas Huamán registró una serie de sitios como Pillucho, Lawirasqa, entre otros; estos estarían conformados por estructuras circulares asociados a cerámica del estilo Arqalla; por lo tanto, estos serían del Periodo Intermedio Tardío y estarían asociados a los grupos denominados chankas, quiénes «son contemporáneos e inmediatamente anterior a los Incas» (Lumbreras, 1959: 237).

Guzmán (1959) en 1958 realizó trabajos de investigación en Vilcas Huamán registrando sitios arqueológicos de diferentes periodos que van desde el Periodo Inicial hasta el Horizonte Tardío. Los sitios del Periodo Intermedio Tardío se encontraron asociados a cerámica del estilo Arqalla. Uno de los principales aportes de Guzmán es que propuso el primer cuadro cronológico señalando a la cerámica Arqalla como perteneciente al Período Intermedio Tardío.

Chahud (1966) en 1965 realizó trabajos de excavación y prospección en Vilcas Huamán, resultado de las excavaciones posicionó estratigráficamente la cerámica del estilo Arqalla como posterior a Wari y previo a la ocupación inca. Incluso encontró cerámica del estilo Arqalla asociada a cerámica inca, lo cual lo llevó a plantear su contemporaneidad.

Ochante (2001) entre los años 1990 y 2000 realizó trabajos de prospección en el distrito de Concepción (Vilcas Huamán), producto de ello registró una serie de sitios perteneciente tanto al Periodo Intermedio Tardío y Horizonte Tardío. Este investigador es el único referente en torno al sitio arqueológico Llaqtaqasa, lo describió de manera general clasificándolo como un sitio de habitación conformado por un sistema de 
estructuras circulares asociados a corrales distribuidos en la cima de un cerro y protegido por murallas pertenecientes al Periodo Intermedio Tardío. Además de Llaqtaqasa, registró los sitios de Huanacauri, Maucallaqta, Churcurumi, Urquncorral, Pucara, Llaqtapata, Qullqui Pedro, Tuirunga y Muela Qasa, estos estarían conformados por estructuras circulares hechas a base de piedras unidas y distribuidas de «manera desordenada» a lo largo de la cima de los cerros, contarían con un sistema de murallas, corrales y zanjas, también registró sitios de inhumación y andenes.

Vaquerizo (2003), realizó un reconocimiento en San Antonio de Cocha (distrito de Huambalpa), Castro (2007) en la cuenca del río Itanayuq (Pujas), Quijano (2010) entre las quebradas de Saurama y Urpay Puquio (distrito de Carhuanca), Mendoza (2010) en la margen izquierda de los ríos Yanamayu y Pampas pertenecientes al distrito de Vilcas Huamán, Quispe y Rojas (2010) en la margen izquierda del río Qatunmayo (distrito Vischongo) y Mendoza (2014) al sureste del distrito de Vilcas Huamán.

Todos ellos han registrado sitios desde el Periodo Inicial hasta el Horizonte Tardío, concluyendo que los sitios del Periodo Horizonte Medio estarían ubicados en las partes bajas, cerca de los valles, asociados a cerámica del estilo Huamanga y a un sistema de andenerías. Respecto a los sitios del Periodo Intermedio Tardío, estarían ubicados en las partes altas (cima de los cerros) asentados, en su gran mayoría, sobre áreas sin ocupaciones previas, algunos asociados a murallas, zanjas, abrigos rocosos, andenes y corrales. Estos se encontrarían compuestos por una serie de recintos circulares de piedras entre 4 a $6 \mathrm{~m}$ de diámetro orientados sin un orden aparente. Para el caso de Pujas, Castro (2007) estableció dos categorías de sitios del Periodo Intermedio Tardío: Pukarás o fortalezas y poblados sin fortificación. El primer tipo estaría caracterizado por su inaccesibilidad, presencia de un sistema defensivo y control de acceso, estos se encuentran compuestos por una serie de recintos circulares entre 4 a $6 \mathrm{~m}$ de diámetros aglutinados entre 4 a 5 alrededor de un espacio (patio) y distribuidos a lo largo de la cima de los cerros, además estarían asociados a corrales, abrigos rocosos (donde se encontraron restos humanos), terrazas agrícolas, murallas defensivas, zanjas, estructuras rectangulares, estructuras circulares pequeñas, entre otros. Por otra parte, los poblados sin fortificación son aquellos que carecen de un sistema de defensa, tienen fácil acceso y se encuentran asociados a terrazas agrícolas.

\section{Metodología}

El asentamiento Llaqtaqasa fue dividido en tres sectores (A, B y C). Esto en base a una serie de ítems como la ubicación topográfica en torno al cerro Llaqtaqasa, la presencia y cantidad de componentes arquitectónicos como los muros concéntricos que separan el sector A del B, entre otros.

Posteriormente se realizó en el sector A y C los dibujos a manos alzada donde se plasmaron la mayoría de componentes y elementos arquitectónicos. Sin embargo, en el sector B se realizó el levantamiento planimétrico y topográfico de todos los componentes arquitectónicos identificados en superficie, esto con la ayuda de una estación total.

Una siguiente etapa consistió en trabajos de prospección sistemática con cobertura total a través de transectos lineales. El primer recorrido tuvo como objetivo describir todos los componentes y elementos arquitectónicos registrados en los distintos sectores, para esto se utilizaron fichas. Dentro de estas se consideraron datos como técnica constructiva, material constructivo, diámetro de recinto, largo y ancho de muros, entre otros. Además, se elaboraron algunos dibujos arquitectónicos y arqueológicos.

El segundo recorrido se centró exclusivamente en el sector B ya que es el más extenso y tuvo como objetivo recolectar información de los objetos muebles (cerámica y artefactos líticos) asociados a las diferentes estructuras, este no contempló recolección ni traslado de material; por lo tanto, todo el registro gráfico y escrito se realizó in situ.

\section{Ubicación del sitio arqueológico Llaqtaqasa}

El sitio arqueológico Llaqtaqasa tiene una extensión de 29.27 hectáreas, está ubicado entre los anexos de Astanya y Pirhuabamba, pertenecientes al distrito de Concepción, provincia Vilcas Huamán, región Ayacucho, entre las coordenadas UTM: N 8497552 / E 621655. Se encuentra en la margen izquierda del río Pampas, a $10.7 \mathrm{~km}$ al noreste de la ciudad de Vilcashuamán (ver figura 1), $1.2 \mathrm{~km}$ al sur del centro 


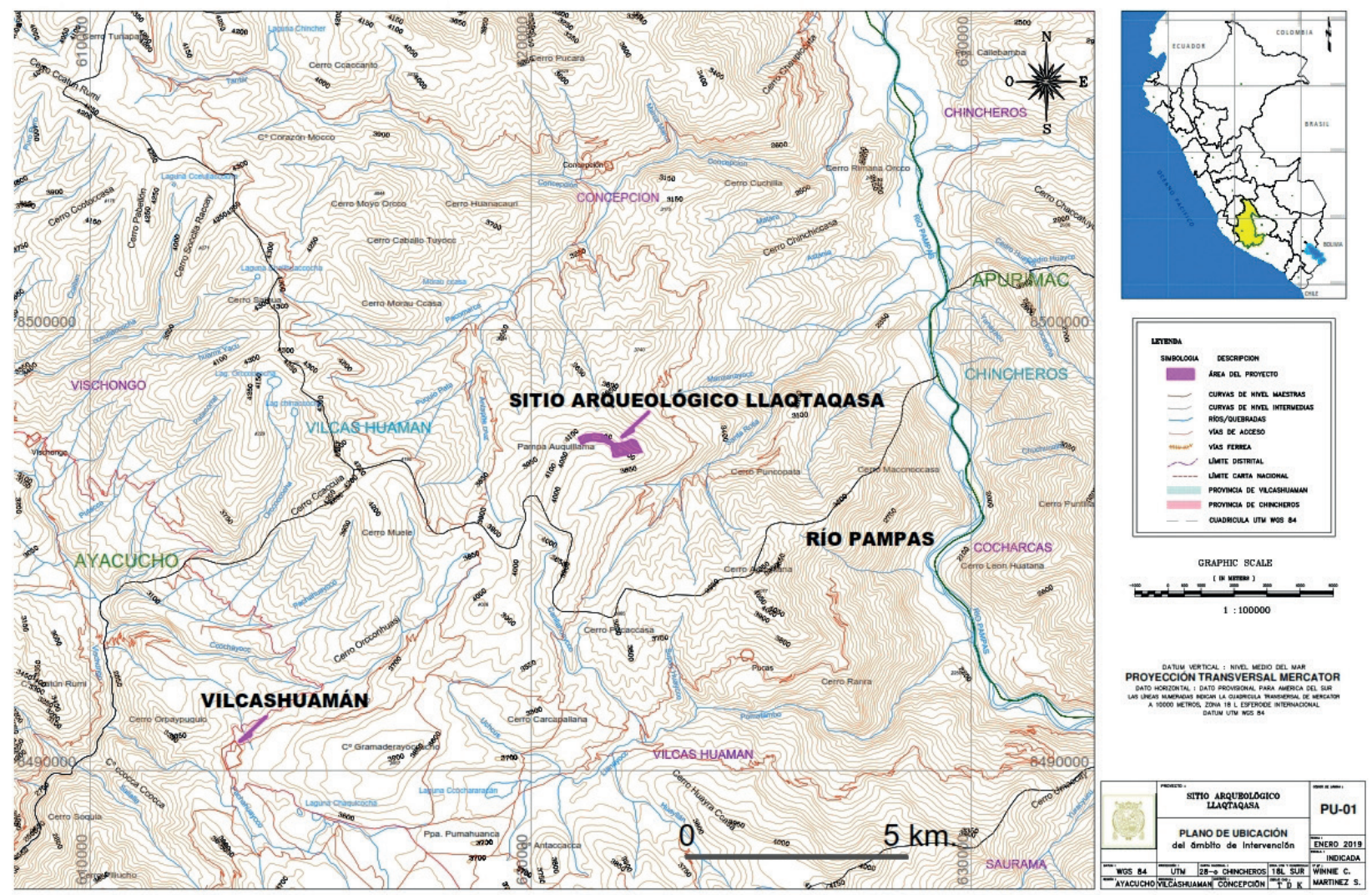

Figura 1. Vista de ubicación del sitio arqueológico Llaqtaqasa en relación a la ciudad de Vilcashuamán y al río Pampas (2019).

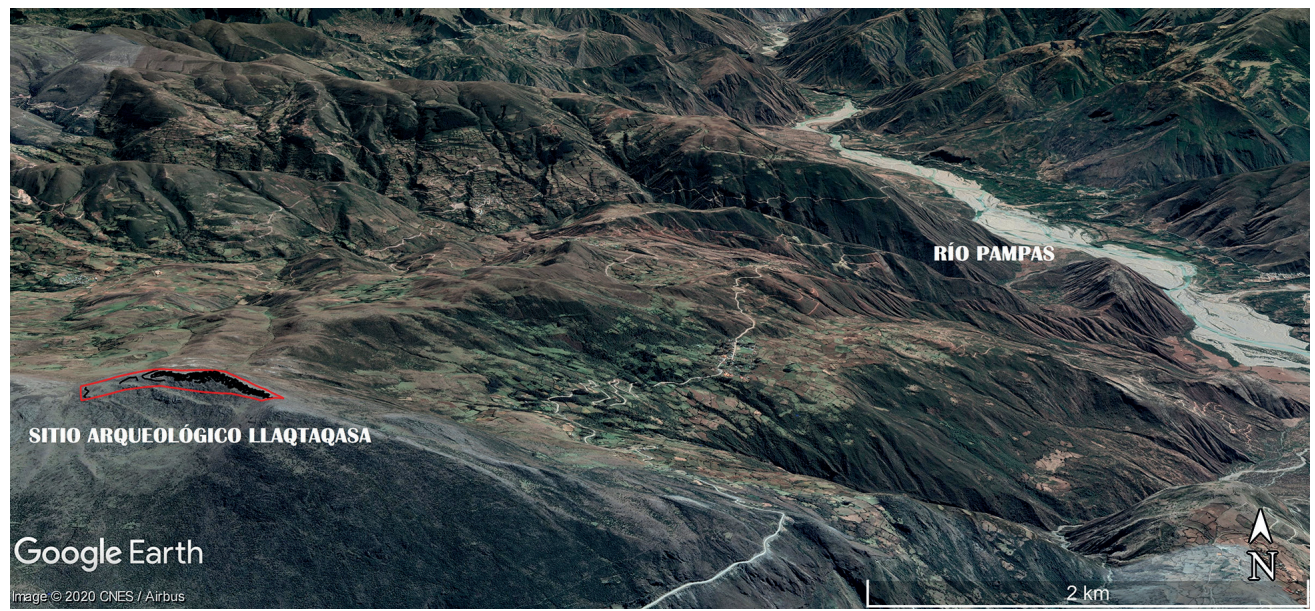

Figura 2. Vista de ubicación del sitio arqueológico Llaqtaqasa en relación con el río Pampas, nótese la geomorfología del área circundante (fuente: Google Earth, 2020).

poblado de Astanya, $2.2 \mathrm{~km}$ al este del centro poblado de Pirhuabamba y $7 \mathrm{~km}$ al oeste del río Pampas.

Se emplaza en la cima y ladera de un promontorio rocoso conocido por los pobladores como Llaqtaqasa (denominada pampa Auquillama en la carta nacional Chincheros 28 O). Este promontorio se encuentra conformado por dos colinas orientadas de sureste a noroeste, delimitadas a su vez por las quebradas de Antapite Cruz (lado oeste) y Manzanayocc (lado este).
El relieve es escarpado y forma parte del grupo de rocas Pucará constituido por calizas gris azulinas en bancos medios a gruesos con nódulos de chert (Sílex Pedernal) formado en el periodo Triásico Superior $(228.0 \pm 2$ hasta 199.6 \pm 0,6 millones de años) de la era Mesozoica (Carta Geológica INGEMMET Chincheros «28 O»).

Llaqtaqasa se emplaza entre los 4000 a 4150 msnm por lo que, según Pulgar Vidal (1996), se ubicaría en el límite entre la región Suni y Puna. 


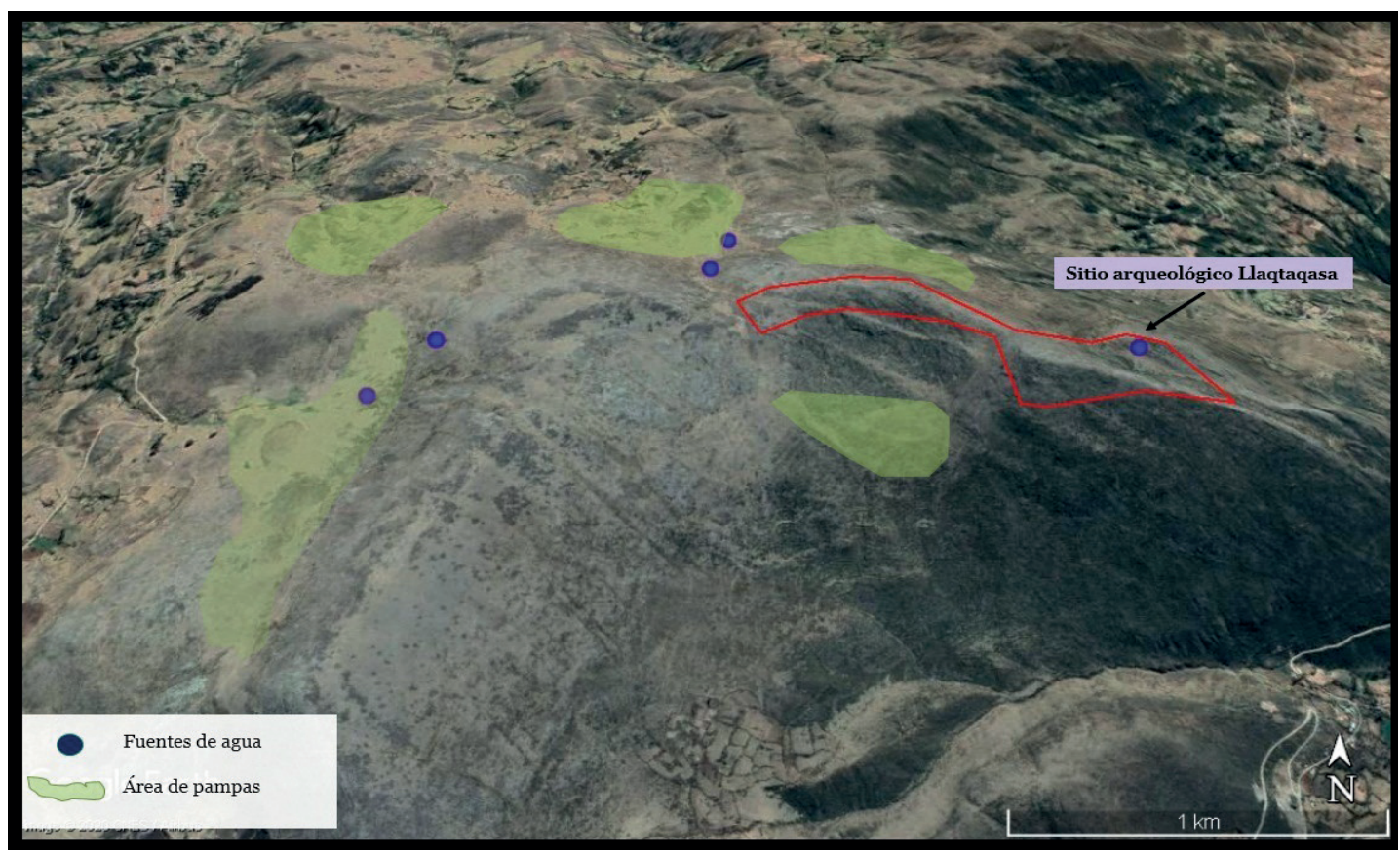

Figura 3.

Vista de

las áreas

de pampas

$y$ fuentes

de agua

ubicados

en los

alrededores

del sitio

arqueológico

Llaqtaqasa

(fuente:

Google

Earth, 2020).

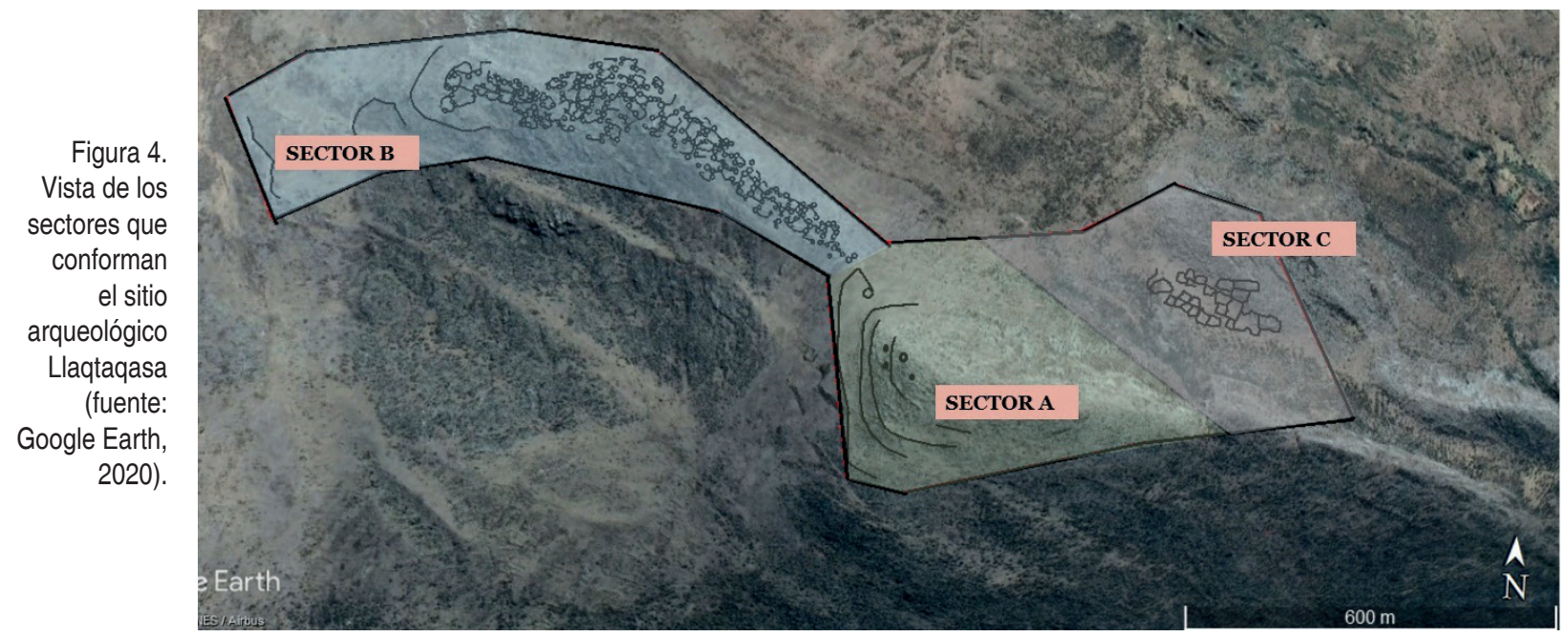

La vegetación predominante es la champa, pajonales como el ichu, puyas de Raimondi, entre otros. Respecto a la fauna están las vizcachas, aves como el halcón, perdiz y aves de laguna. Entre los productos límites se tiene a la papa, cebada, maca, etc.

El sitio arqueológico se ubica en la cima de un afloramiento rocoso con un relieve accidentado (ver figura 2), sin embargo, en los alrededores (300 a $500 \mathrm{~m}$ ) se observa grandes áreas de pampas ubicadas sobre los $3500 \mathrm{msnm}$ asociados a fuentes de agua (ver figura 3). Actualmente la principal actividad realizada en esta zona es el pastoreo, la agricultura no es practicada debido a la caída de constantes heladas que dañan los cultivos.
Llaqtaqasa, proviene de dos vocablos quechuas: Llaqta (pueblo) y Qasa (helada) que hace referencia a un pueblo donde cae constantemente helada.

Durante el Periodo Colonial esta zona perteneció al curato de Pacomarca (Carabajal, 1965 [1881]) del corregimiento de Vilcashuamán. Posteriormente, a partir del año 1603, estas tierras formaron parte de la hacienda Astania ${ }^{1}$ pertenecientes en un primer momento al padre jesuita Juan de Ayala.

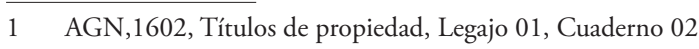




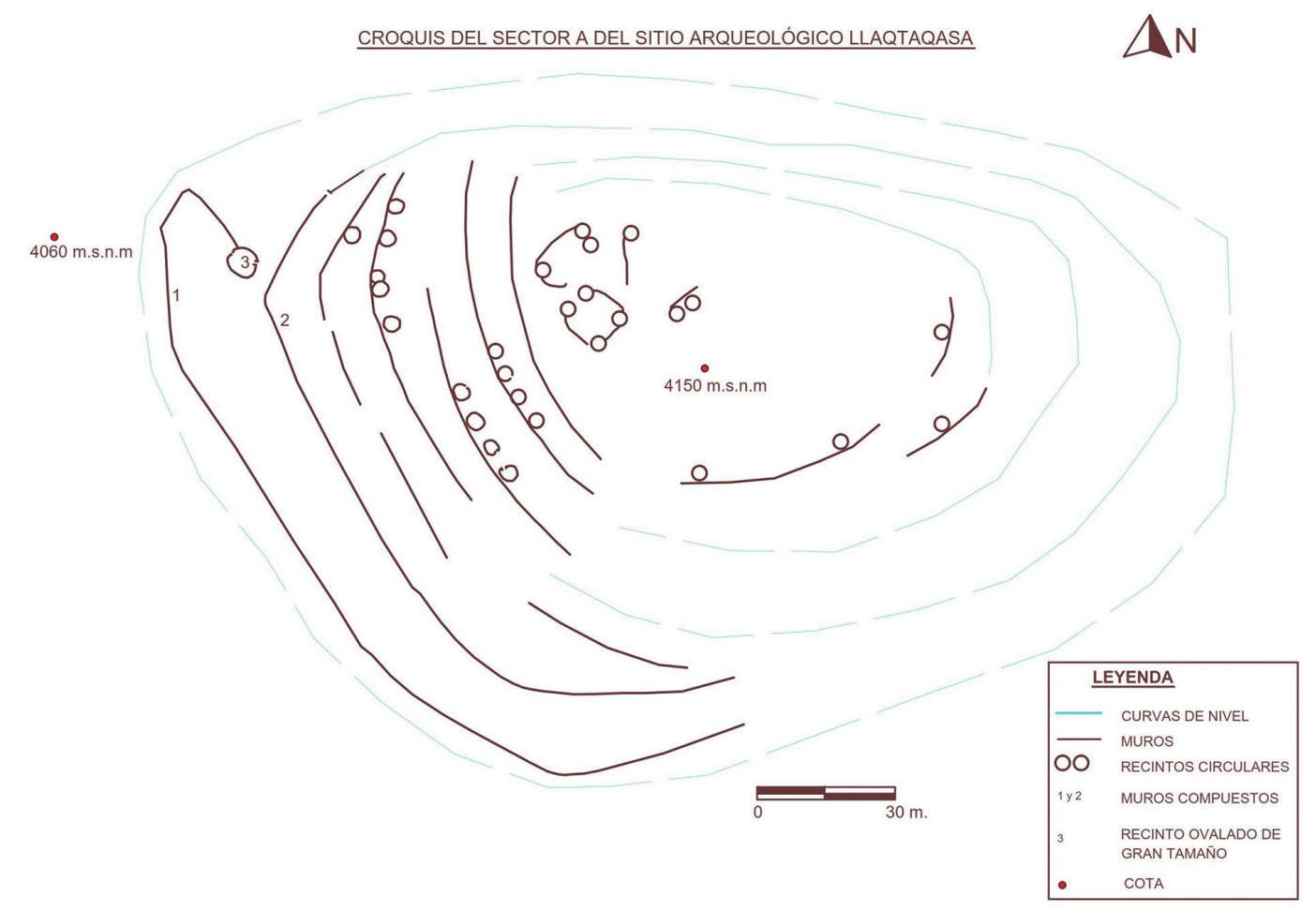

Figura 5.

Croquis

del sector

A del sitio

arqueológico

Llaqtaqasa.

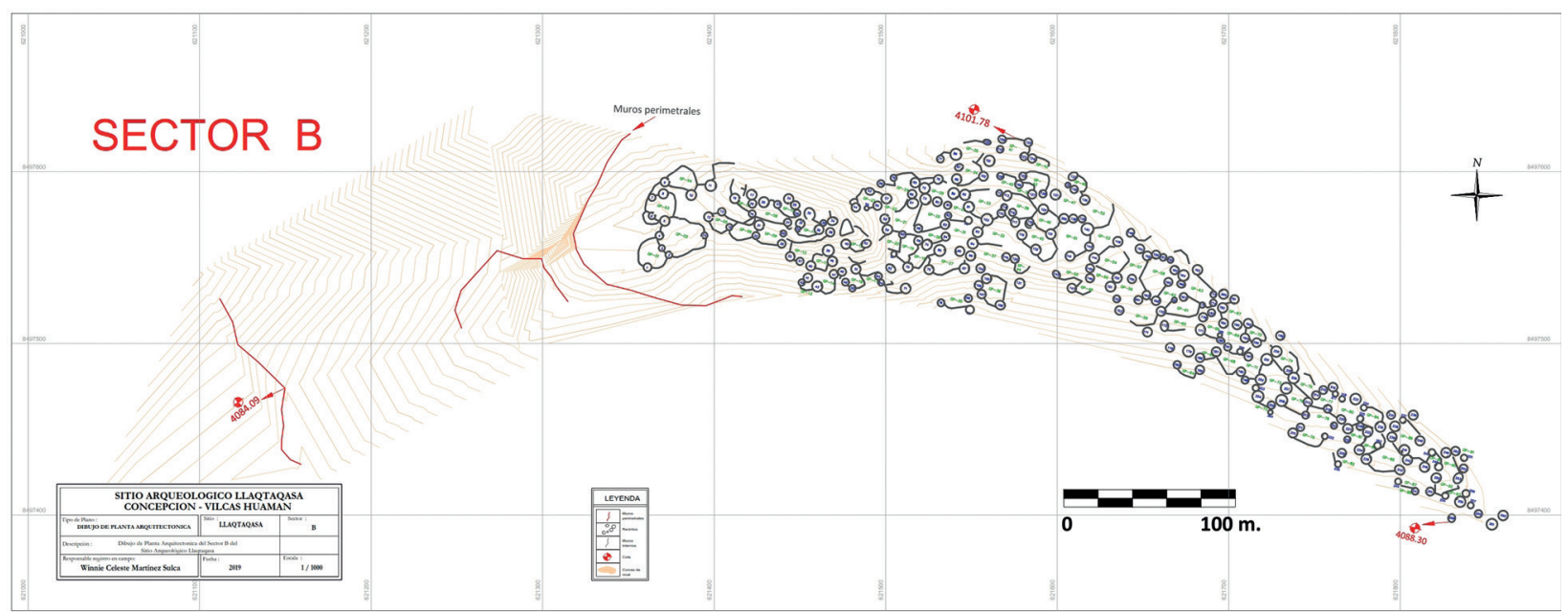

Figura 6. Plano del sector B del sitio arqueológico Llaqtaqasa.

\section{Sectorización}

El asentamiento Llaqtaqasa fue dividido en tres sectores ( $\mathrm{A}, \mathrm{B}$ y $\mathrm{C}$ ), esto en base a la ubicación, emplazamiento y presencia de componentes arquitectónicos como muros compuestos (ver figura 4). En campo se realizó el croquis de los tres sectores, así como la descripción de todos los componentes arquitectónicos y materiales arqueológicos muebles en superficie.
Específicamente en el sector B se realizó el levantamiento planimétrico y topográfico de todas las estructuras con la ayuda de una estación total.

\subsection{Sector $A$}

El sector A (ver figura 5) se encuentra ubicado en la cima y ladera de la segunda colina del cerro Llaqtaqasa, específicamente al lado sureste del asentamiento, co- 


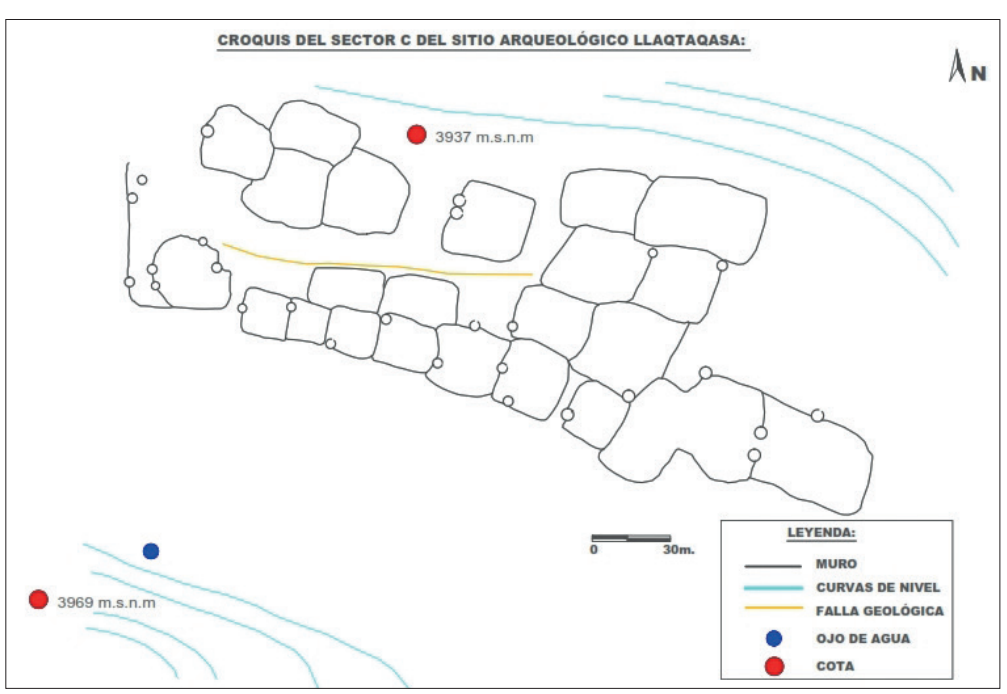

Figura 7. Croquis del sector $\mathrm{C}$ del sitio arqueológico Llaqtaqasa.

linda por el noroeste con el «sector B», separado de este último por medio de una serie de muros compuestos. Esta colina es la más alta ya que tiene una altitud de $4150 \mathrm{msnm}$ (ver figura 8) y una forma tronco piramidal, además de una extensión de $380 \mathrm{~m}$ de largo (este a oeste) y $220 \mathrm{~m}$ de ancho de norte a sur, haciendo un total aproximado de 8.36 ha.

Este sector se encuentra conformado por una serie de muros compuesto en forma de «U» que bordean la parte inferior y media de dicha colina, sobre estas se registraron una serie de recintos de planta circular organizados de manera lineal, mientras que en la cima los recintos se encuentran organizados en torno a un patio, este último a su vez se encuentra delimitado por muros bajos doble cara.

En superficie se halló gran cantidad de material cerámico correspondiente a ollas, cántaros, platos, etc. Además de material lítico como batanes, morteros, manos de moler y porras.

\subsection{Sector $B$}

El sector B (ver figura 6) se encuentra ubicado en la cima del cerro Llaqtaqasa, al noroeste del asentamiento, presenta una orientación de este a oeste ocupando una superficie relativamente ondulada donde predominan afloramientos rocosos abarcando un total de 12.48 ha. Este sector es el más denso y numeroso se ubica entre los 4091 a 4116 m.s.n.m.

$\mathrm{Al}$ interior se registraron alrededor de 266 recintos de planta circular (en su gran mayoría) y ovalada con un diámetro que varía entre 2.5 a $6 \mathrm{~m}$, los muros presentan doble hilera y tienen un ancho que varía entre 0.6 a $0.9 \mathrm{~m}$, el paramento interior y exterior se encuentran elaborados en base a piedras calizas semi canteadas de $0.2 \times 0.3 \times 0.4 \times 0.5 \times 0.6 \times 0.7 \mathrm{~m}$, siendo las piedras más grandes colocadas en las bases de los muros. Estas piedras fueron colocadas unas tras otras exponiendo el lado más plano, carecen de mortero y enlucido.

Estas estructuras son de una sola pieza y nivel, carecen de hornacinas y fueron erigidos directamente sobre roca madre, el piso no presenta revestimiento (ver figuras 10 y 11). Algunas de ellas han sido construidas sobre terrazas en la periferia del sector donde el terreno es relativamente inclinado. Los accesos siempre están orientados al interior de los patios, estos a su vez se encuentran delimitados por muros bajos doble cara, este tipo de organización espacial corresponde a los «Grupo-patio» (ver figura 12) definido para la sierra central por T-Earle (1987) y desarrollado por D'Altroy y Hastorf (2002).

Sobre la zona medianamente accesible donde no hay obstáculos naturales, exactamente en el extremo oeste del sector $\mathrm{B}$, se registraron 3 muros perimetrales doble cara entre 20 a 100 m de longitud, estas delimitan toda la parte oeste del sector limitando el acceso hacia el mismo. Todos estos se encuentran construidos directamente sobre roca madre, están conformados por piedras no trabajadas poliédricas de $0.4 \times 0.6 \times 0.8 \times 1 \times 1.2 \mathrm{~m}$, carecen de mortero y enlucido, no forman hileras, forman un aparejo de tipo rústico, presentan un perfil natural, el núcleo se encuentra compuesto por piedras no trabajadas rellenas con cascajo grueso de 3 a $6 \mathrm{~cm}$. El ancho del muro varía entre 0.9 a $1.1 \mathrm{~m}$, la altura conservada va desde 0.05 a $1.4 \mathrm{~m}$. Los muros tienen diversas formas que van desde la recta a semiovalada.

La superficie del espacio entre los muros perimétricos se encuentra conformada por afloramientos rocosos y tiene una pendiente descendente de este a oeste, en el interior no se registró ningún tipo de estructura arquitectónica.

En superficie se halló gran cantidad de material cerámico correspondiente a ollas, cántaros, platos, vasos, etc. Además de material lítico como batanes, morteros, manos de moler, rompeterrones, entre otros. 


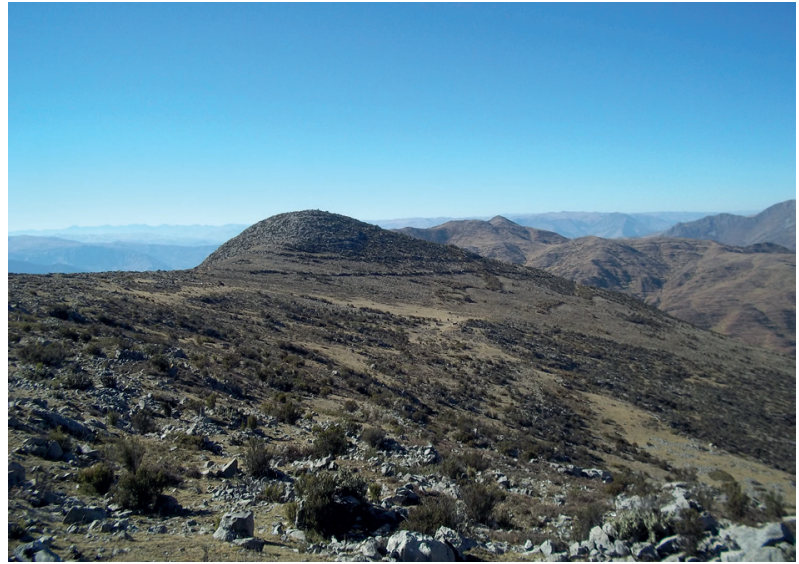

Figura 8. Vista del sector A del sitio arqueológico Llaqtaqasa, nótese los muros compuestos en la ladera

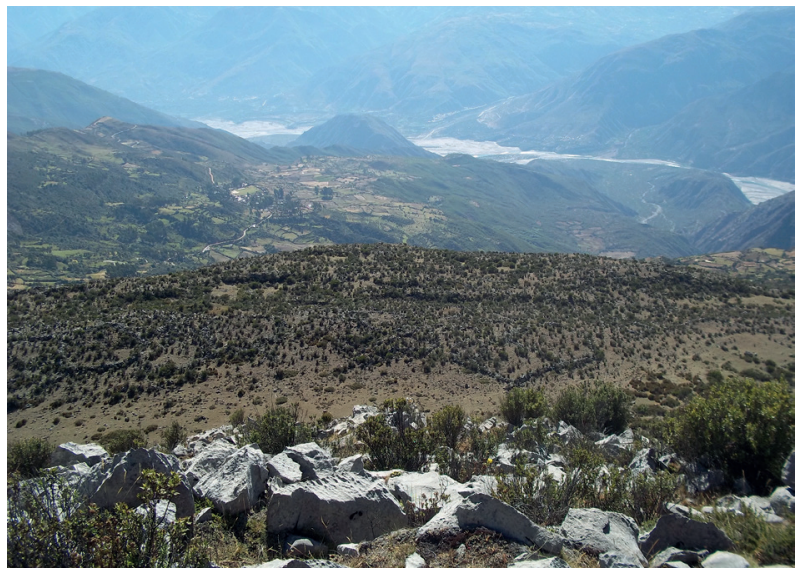

Figura 10. Vista general del sector B.

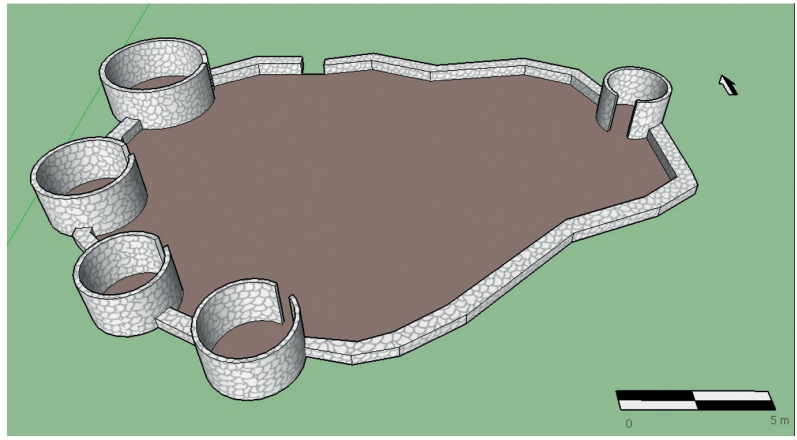

Figura 12. Vista de isometría de «Grupo-patio» registrado al interior del sector B del sitio arqueológico Llaqtaqasa.

\subsection{Sector $C$}

El sector C (ver figura 7) se encuentra ubicado al pie del cerro Llaqtaqasa, en la zona conocida por los pobladores como "Llaqtaqasa Cucho", al noreste y en la parte baja del asentamiento, se emplaza en una zona

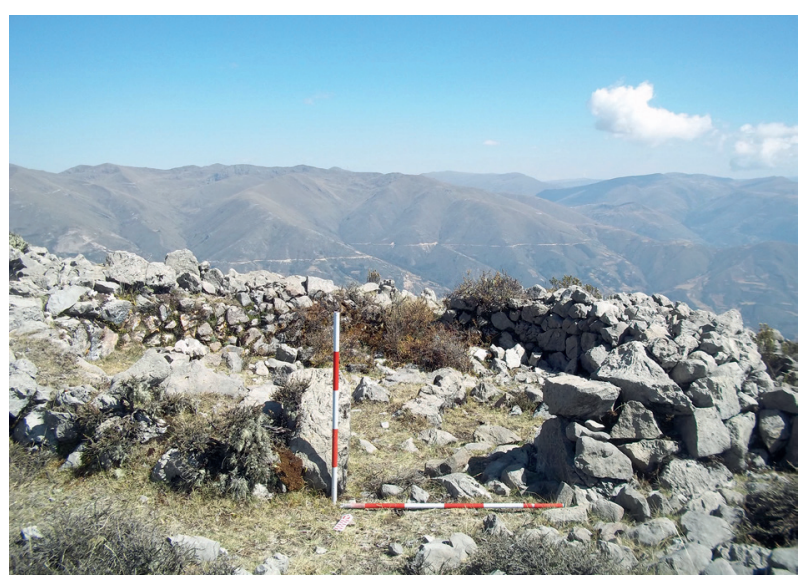

Figura 9. Vista de recinto de planta circular al interior del sector B.

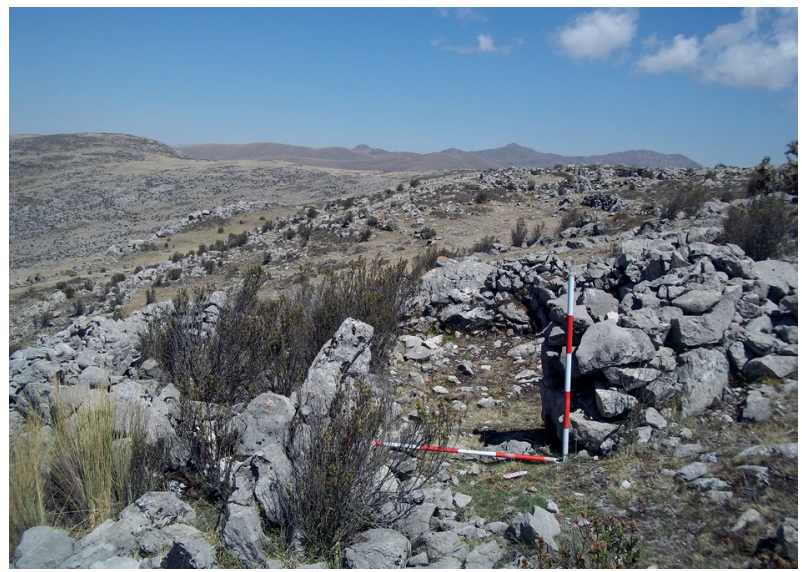

Figura 11. Vista de recinto de planta circular que forma parte del sector B del sitio arqueológico Llaqtaqasa.

relativamente plana compuesto por afloramientos rocosos sobre los $3948 \mathrm{msnm}$, tiene una extensión de $389 \mathrm{~m}$ de largo de este a oeste y $187 \mathrm{~m}$ de ancho de norte a sur haciendo un total de 7.27 ha.

$\mathrm{Al}$ interior se registró una serie de corrales de forma irregular de gran tamaño de 20×30 m organizados de forma celular asociados a recintos de planta circular y a fuentes de agua (ver figura 9). Los muros que lo conforman son de piedras no trabajadas poliédricas de diferentes tamaños: $0.6 \times 0.9 \times 1.1 \mathrm{~m}$, no presentan mortero ni enlucido, son cara tosca, no presentan hileras, son de doble cara y tienen una altura conservada que varía entre 0.3 a $1 \mathrm{~m}$ aproximadamente.

La ubicación de este sector es estratégica ya que debido a su altitud y a su topografía la sensación de frío es menor a comparación de los demás sectores, además se ha registrado un ojo de agua de regular tamaño en el extremo sur. 


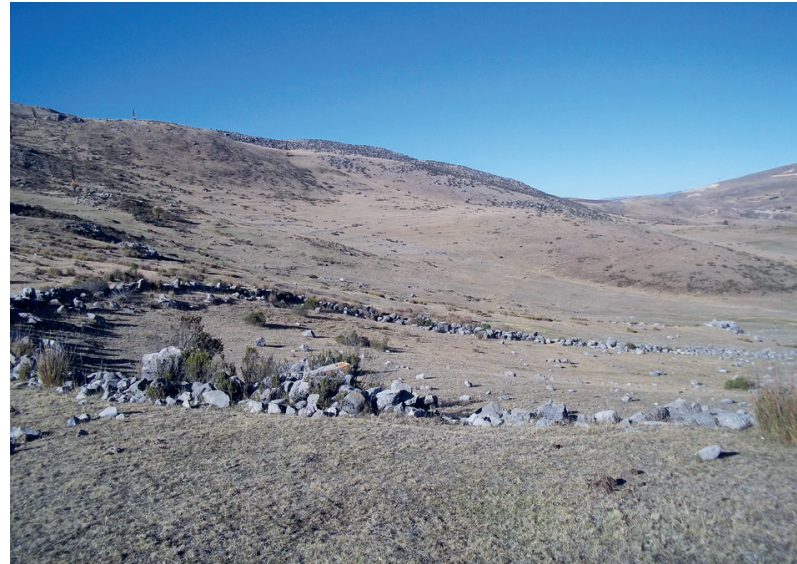

Figura 13. Vista de corrales ubicados en los alrededores del sitio Llaqtaqasa.

\section{Estructuras arquitectónicas aledańas (corrales $y$ andenes)}

Alrededor del sitio arqueológico Llaqtaqasa, a una distancia entre $0.4-1.2 \mathrm{~km}$, se registró una serie de corrales de gran tamaño (ver figura 13), todas ellas ubicadas en zonas relativamente planas por debajo de los 4000 m.s.n.m asociadas a ojos de agua y afloramientos rocosos. En general, estos corrales se organizan espacialmente de forma celular y su forma es variable desde semicircular, ovalada y cuadrangular. Se encuentran elaboradas a base de piedras no trabajadas angulosas de $0.3 \times 0.5 \times 0.7 \mathrm{~m}$, no presentan mortero ni enlucido, no presentan hileras, son de cara tosca, el ancho del muro varía entre 0.3 a $0.5 \mathrm{~m}$ y se encuentra compuesto por una sola hilada de piedra, la altura conservada varía entre 0.3 a $1 \mathrm{~m}$. Algunos de estos se encuentran asociados a recintos circulares de $4 \mathrm{~m}$ de diámetro.

Además, en la parte baja del sitio (500 $\mathrm{m}$ al este) se identificó una serie de andenes (ver figuras 14 y 15) orientados de norte a sur abarcando un total de 0.9 ha, estos se encontraban asociados a recintos de planta circular.Los muros de contención se encuentran elaboradas a base de piedra no

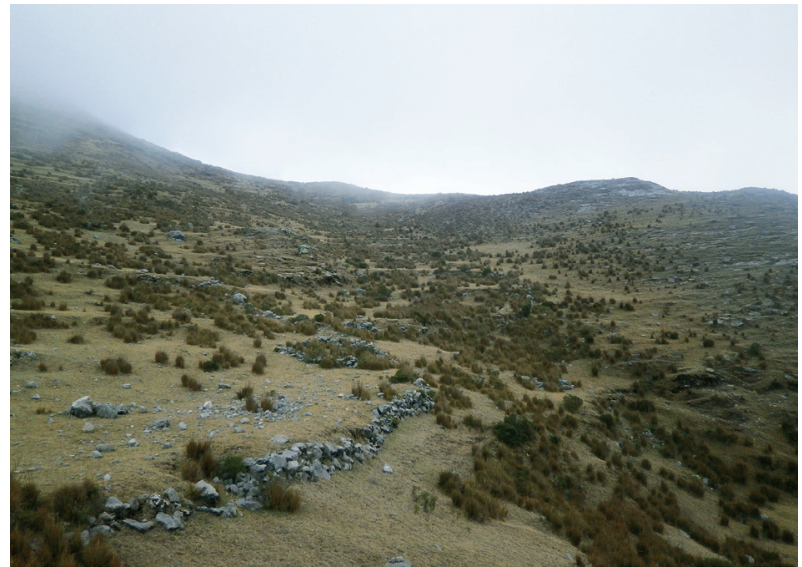

Figura 14. Vista de andenes ubicados al pie del sitio arqueológico Llaqtaqasa.

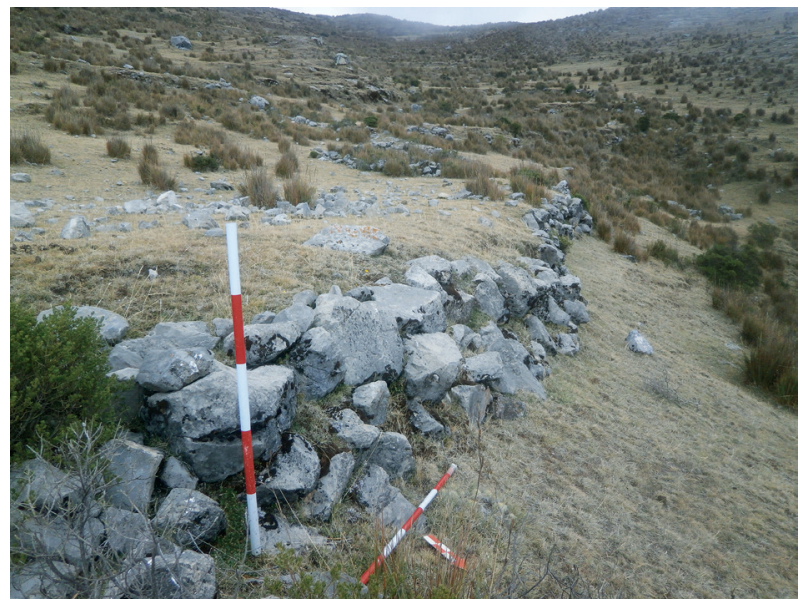

Figura 15. Vista lateral de muros de contención que forman parte de los andenes ubicados al pie del sitio arqueológico Llaqtaqasa.

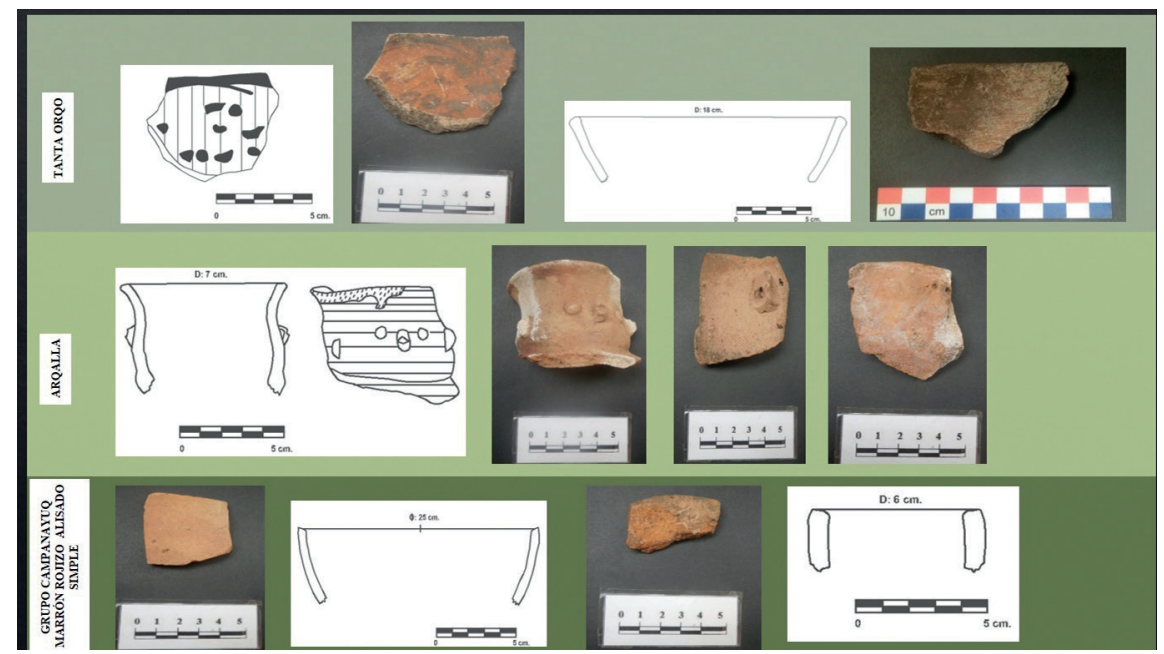

Figura 16. Vista de tipos y grupos de cerámica identificados en superficie al interior del sitio arqueológico Llaqtaqasa. 
trabajadas poliédricas de $0.2 \times 0.3 \times 0.4 \times 0.5 \mathrm{~m}$, carecen de mortero y enlucido, las piedras son de cara tosca, no forman hileras, presenta una orientación de este a oeste con cara al norte, el ancho de los muros varía entre 0.6 a $0.7 \mathrm{~m}$, la altura conservada entre 0.2 a $1 \mathrm{~m}$ y el largo entre 50 a $70 \mathrm{~m}$.

\section{Materiales muebles en superficie}

\subsection{Cerámica}

En los tres sectores se registró gran cantidad de fragmentos de cerámica en superficie; sin embargo, en este punto presentaremos los datos del análisis realizado dentro del sector B. Hay que recalcar que este no contempló traslado ni recojo de material.

En total se analizaron 111 fragmentos, de los cuales 89 corresponden a cerámica diagnóstica y 22 a no diagnóstica. Basados en Ravines (1989), en esta sección estamos considerando dos categorías de vasijas: abiertas y cerradas, las primeras son aquellas cuyo diámetro de boca es proporcionalmente equivalente o mayor a su altura, las segundas son aquellas cuyo diámetro de su boca es menor a su altura.

De los 89 fragmentos de cerámica diagnóstica, $23(26 \%)$ corresponden a vasijas abiertas y $66(74 \%)$ a vasijas cerradas.

Vasijas abiertas. Al interior de esta categoría se identificaron 20 platos y 3 vasos, que corresponden al $22 \%$ y $3 \%$ respectivamente del total de la muestra.

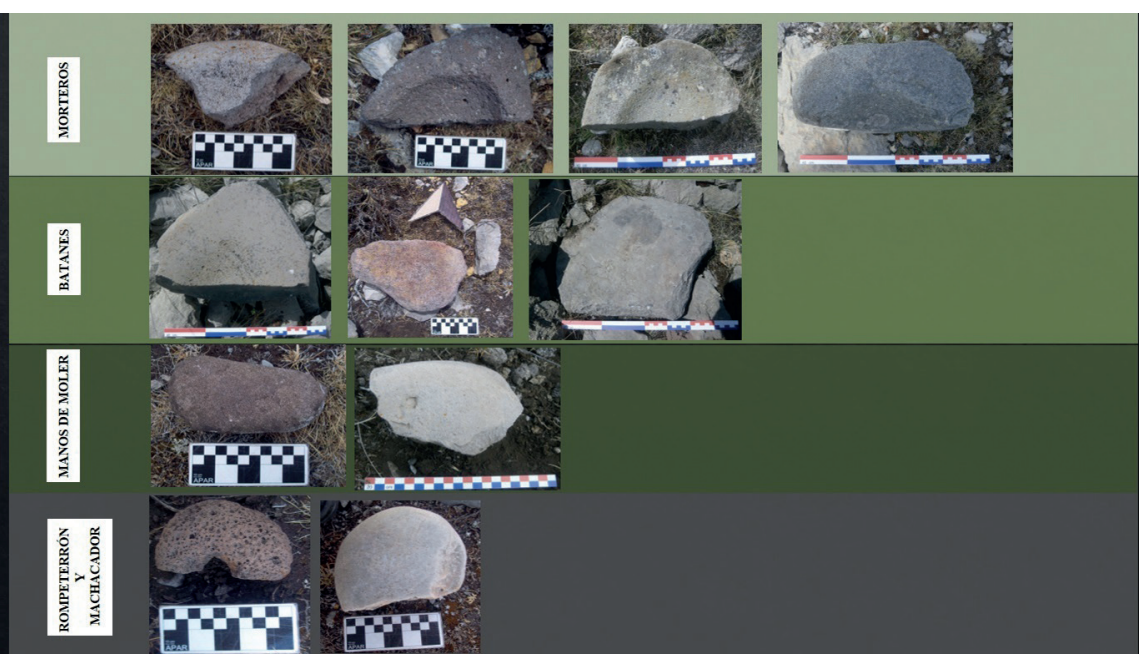

Figura 17. Vista de materiales líticos hallados en superficie en el sitio arqueológico Llaqtaqasa.
Vasijas cerradas. Al interior de esta categoría se identificaron 55 cántaros, 10 ollas y 1 botella que corresponde al $62 \%, 11 \%$ y $1 \%$ respectivamente del total de la muestra.

Se pudo observar que muchas de estas vasijas guardan relación con los tipos y grupos establecidos para esta zona perteneciente al Periodo Intermedio Tardío (ver figura 16). Por ejemplo, 19 fragmentos de cerámica guardan relación con el grupo Campanayuq marrón rojizo alisado simple identificado por Mendoza (2010) en el sitio arqueológico Campanayuq Rumi, ubicado en el distrito de Vilcas Huamán. En esta la pasta es de color naranja, presenta como inclusiones cuarzo, feldespato y mica, el tratamiento interior y exterior de la superficie es un alisado tosco con estrías, carece de decoración. Dentro de esta tenemos vasos, ollas, platos y cántaros.

Por otro lado, 43 fragmentos comparten ciertas características con el tipo Arqalla (González et al, 1987). En estas la pasta tiene un color que va de naranja a naranja rojizo, presentan como inclusiones cuarzo, feldespato y mica, el tratamiento interior y exterior de la superficie presenta un alisado medio con engobe de colores rojizo, naranja rojizo entre otros. Además, algunos fragmentos presentan decoración en base a aplicaciones tipo cara gollete y otros tienen en el borde del labio un engobe diluido de color rojizo. Dentro de esta tenemos vasos, ollas, platos y cántaros.

Por último, tres fragmentos guardan relación con el tipo Tanta Orqo (González et al, 1987). La pasta es de color blanco grisáceo, presentan como inclusiones cuarzo, feldespato y mica, el tratamiento interior y exterior corresponde a un alisado fino con engobe exterior de color marrón rojizo. Un fragmento presenta decoración en la superficie exterior en base a pintura de color negro que forman líneas y puntos.

\subsection{Lítico}

El análisis de los artefactos líticos hallados en superficie al interior del sector B se realizó in situ sin alterar 
el contexto original. En total se registraron 17 artefactos líticos (ver figura 17), de los cuales 7 (41\%) corresponden a morteros, $6(35 \%)$ a batanes, 2 (11.7\%) manos de moler, 1 rompeterrón $(5.8 \%)$ y 1 machacador (5.8\%). La gran mayoría de estos fueron elaborados en base a piedras tipo andesita y granito.

Además, en otros sectores se identificó fragmentos de porras elaborados con granito.

\section{Discusión preliminar}

En base a las características arquitectónicas, los componentes, el emplazamiento, los materiales hallados en superficie, como la cerámica del grupo Campanayuq marrón rojizo alisado simple (Mendoza, 2010), el tipo Arqalla y Tanta Orqo (González et al, 1987) y la comparación con otros asentamientos registrados por Lumbreras (1959), Guzmán (1959), Chahud (1966), Ochante (2001), Vaquerizo (2003), Quijano (2010), Mendoza (2010), Mendoza (2014), entre otros. Podemos mencionar que el sitio arqueológico Llaqtaqasa fue construido durante el Periodo Intermedio Tardío (1100-1400 D.C).

Probablemente durante el Periodo Horizonte Tardío fue abandonado ya que no encontramos evidencias claras de la presencia inca como sí se han identificado en otros asentamientos tales como Pillucho y Lawirasqa (Mendoza, 2010), ubicados cerca de la ciudadela inca de Vilcashuamán. En estos últimos se registró cerámica del estilo inca local, conopa en forma de llama y estructuras cuadrangulares con hornacinas de forma trapezoidal.

Por otro lado, podemos seńalar que el sitio arqueológico Llaqtaqasa presenta tres sectores claramente diferenciados y separados espacialmente, donde señalamos que los sectores A y B corresponderían al área doméstica (en base a la gran cantidad de cerámica doméstica como platos, ollas, cántaros, vasos y artefactos líticos como morteros, manos de mortero, batanes, rompeterrones, entre otros), siendo este último el área más extensa conformada por un denso número de recintos circulares. En el extremo oeste se registraron tres muros perimétricos o también llamados murallas que tenían como función restringir el acceso hacia el mismo.
El sector A es el área más pequeña, mejor posicionada y restringida de todos los sectores. Este último debido a que se encuentra rodeado por una serie de muros concéntricos de regular altura que limitan el acceso a los habitantes del sector B. Este podría ser tal vez el espacio doméstico de un grupo familiar con cierto grado de jerarquía.

Por último, el sector C está conformado por una serie de corrales asociados a recintos circulares, estas estarían destinada a la crianza de camélidos. Esta actividad se habría realizado con normalidad debido a que el área cuenta con abundantes zonas de pampas, abrigos rocosos y ojos de agua ubicados en un perímetro de $1.5 \mathrm{~km}$; por otro lado, la sensación de frío en este sector es menor ya que se encuentra protegido por afloramientos rocosos. Este patrón de ubicación de corrales también es registrado por Lavallée y Julien (1983) para los sitios Astos, mencionan que casi siempre los corrales «estaban protegidos del viento y situadas en dirección al punto de agua más cercano» (Lavallée y Julien, 1983:58). Los recintos circulares asociadas a los corrales corresponderían a viviendas para los pastores, esto en base a la gran cantidad de cerámica de función doméstica hallada en superficie.

No se ha identificado hasta el momento otro tipo de estructuras arquitectónicas como recintos de planta rectangular, plazoletas o estructuras funerarias.

La ubicación de Llaqtaqasa fue estratégica, ya que el área cuenta con abundantes zonas de pampas, fuentes de agua y abrigos rocosos, haciendo fácil el desarrollo de actividades productivas como la crianza de camélidos y cultivo de productos propios de la zona, evidencia de esto último están los andenes ubicados al pie del sitio. Por otro lado, los afloramientos rocosos presentes han servido como canteras, desde donde se han extraído las piedras para la construcción de los recintos circulares y otros componentes arquitectónicos.

Además, el sitio tiene un dominio visual de $360^{\circ}$ y un control total del área circundante, desde este punto podemos observar el río Pampas (actual límite con la región de Apurímac), el apu Ccarhuarazo (provincia de Lucanas), el nevado Salkantay (región Cusco) y otros sitios arqueológicos del Periodo.

Por otro lado, el asentamiento tiene todas las características señaladas por Castro (2007) respecto a un pukará o fortaleza, ya que se encuentra ubicado en un lugar estratégico de carácter inaccesible a una alti- 
tud superior a los $4000 \mathrm{msnm}$ rodeado por murallas y zonas de corrales.

En Llaqtaqasa los recintos de planta circular, sobretodo en el sector $\mathrm{B}$, se organizan entre una a seis alrededor de un espacio común denominado "patio». Este último a su vez se encuentra delimitado por muros bajos doble cara que han sido construidos en áreas donde los edificios no están contiguos sino ligeramente espaciados, este tipo de organización corresponde a los "Grupos-patio» registrado para el valle del Mantaro, región Junín (D'Altroy y Hastorf, 2002). Estos tienen una configuración espacial cerrada donde los recintos y patios, además de ser los principales componentes, forman espacios cerrados restringiendo relativamente la visual y el acceso a los vecinos del área colindante. Además, podemos señalar como propuesta que los "Grupos-patio» en Llaqtaqasa, al igual que para los sitios Wanka (D’Altroy y Hastorf, 2002) podría ser el reflejo de un tipo de organización y economía a nivel familiar.

Por último, este tipo de organización espacial no ha sido registrado previamente en otros sitios del periodo dentro del territorio de Vilcas Huamán, es por ello que consideramos importante ahondar más sobre este tema a fin de entender la lógica social detrás de este tipo de construcciones.

\section{Conclusiones}

El sitio arqueológico Llaqtaqasa fue construido durante el Periodo Intermedio Tardío, esto en base a la cerámica hallada en superficie, las características y componentes arquitectónicos, el emplazamiento y ubicación. Presenta tres sectores claramente diferenciados donde el sector A y B corresponderían al área doméstica, siendo el sector $\mathrm{B}$ el área más densa y extensa, además de presentar tres muros perimetrales que tenían como función restringir el acceso hacia el mismo. Por su parte, el sector C está conformada por una serie de corrales asociado a una fuente de agua.

Alrededor del sitio se registraron una serie de corrales y andenes, donde se harían realizadas actividades productivas como la crianza de camélidos y el cultivo de productos propio de la zona.

\section{Agradecimientos}

Mi principal agradecimiento a mis padres Luis Martínez Fuentes y Cristina Sulca Lara, quienes me apoyaron incondicionalmente en el desarrollo de este trabajo. Asimismo, al profesor Jorge Silva Sifuentes por su contante orientación académica en los diferentes procesos de investigación, a mi compañero José Bazán Castillo quien me acompañó y orientó en los trabajos, a mi primo Fredy Sulca Cárdenas, quien se encargó de los trabajos de levantamiento planimétrico y topográfico del sitio. A la Lic. Jacquely Karim Tarazona, quien me apoyó gustosamente con la elaboración de los planos finales, a la arqueóloga Roxana Mendoza Gutiérrez por el apoyo en los trabajos de campo y, por último, al arqueólogo Ronald Mendoza Mendoza por el apoyo con las referencias bibliográficas.

\section{Apéndice: fuentes históricas inéditas}

Archivo General de la Nación (AGN), Lima

Fondo Índice Campesinado-Títulos de Propiedad (1602).

Títulos de las tierras de la hacienda de Astania-VH.

Legajo:01, cuaderno:02, folio: 13 .

\section{Bibliografía}

Carabajal, P. (1965 [1881]). Descripción fecha de la provincia de Vilcas Guaman por el Illustre Señor don Pedro de Carabajal, corregidor y justicia mayor della, ante Xpistobal de Gamboa, escribano de su juzgado, en el año de 1586. En M. Jiménez de la Espada, Relaciones Geográficas de Indias (Volumen 1) (pp. 205219). Madrid: Ediciones Atlas.

Castro, J. (2007). Prospección Arqueológica en la cuenca del río Itanaynq, Pujas-Vilcas Huamán [Informe de prácticas pre profesionales, Universidad Nacional San Cristóbal de Huamanga, Ayacucho].

Chahud, C. (1966). Investigación Arqueológica en Vilcas Huamán. [Informe de investigación, Universidad Nacional San Cristóbal de Huamanga, Ayacucho].

D'Altroy, N. y Hastorf, C. (Ed.) (2002). Empire and Domestic Economy. New York. Boston. London. Moscow: Kluwer Academics Publishers. 
EARLE, T. (1987). Chiefdoms in archaeological and ethnohistorical perspective. Annual Review of Anthropology, (16), 279-308.

González, E. Pozzi-Escot, D. Pozzi-Escot, M. у Vivanco, C. (1987). Los chankas: Cultura material. Laboratorio de Arqueología de la Universidad Nacional San Cristóbal de Huamanga.

Guzmán, C. (1959). Investigaciones Arqueológicas en Vilcas Huamán, departamento de Ayacucho [tesis de bachillerato, Universidad Nacional Mayor de San Marcos, Lima].

LavalléE, D. y Julien, M. (1983). ASTO: Curacazgo prehispánico de los Andes Centrales (1 $1 \mathrm{ra}$ ed.). Lima. Instituto de Estudios Andinos.

Lumbreras, L. (1959). Sobre los Chankas. Actas y Trabajos del II Congreso Nacional de Historia del Perú. 1, 211-242.

Mendoza, E. (2010). Investigaciones Arqueológicas en la margen izquierda de los ríos Yanamayu y Pampas, Vilcas Huamán- Ayacucho. PACHA RUNA 1, UNSCH. 1, 123-162.

Mendoza, E. (2010). Contextos rituales Tardios, en el centro ceremonial Formativo de Campanayuq Rumi, Vilcas Huamán-Ayacucho [tesis de licenciatura, Universidad Nacional San Cristóbal de Huamanga, Ayacucho].
Mendoza, R. (2014). Prospección Arqueológica en el Sureste del Distrito de Vilcas Huamán, Ayacucho [informe de prácticas pre profesionales, Universidad Nacional San Cristóbal de Huamanga, Ayacucho].

Ravines, R. (1989). Arqueología Práctica. (1ra.ed.). Lima: Editorial Los Pinos E.I.R.L.

Ochante, C. (2001). Prospección Arqueológica en el distrito de Concepción-Vilcas Huamán [Informe de prácticas Pre Profesionales, Universidad Nacional San Cristóbal de Huamanga, Ayacucho].

Pulgar, J. (1996). Las ocho regiones naturales, la regionalización transversal (7ma ed.). Lima: PEISA.

Quijano, A. (2010). Prospección Arqueológica entre las quebradas de Saurama y Urpay Puquio, Carhuanca-Ayacucho [informe de prácticas pre-profesionales, Universidad Nacional San Cristóbal de Huamanga, Ayacucho].

Quispe, V. y Rojas, W. (2010). Reconocimiento Arqueológico en la margen izquierda del Río Qatunmayo, VischongoAyacucho [informe de prácticas pre-profesionales, Universidad Nacional San Cristóbal de Huamanga, Ayacucho].

Vaquerizo, F. (2003). Prospección Arqueológica en San Antonio de Cocha, Distrito de Huambalpa, Provincia de Vilcas Huamán-Ayacucho [informe de prácticas pre profesionales, Universidad Nacional San Cristóbal de Huamanga, Ayacucho]. 